AMERICAN

SOCIETY OF

INTERNATIONAL

LAW

\title{
PROCEEDINGS of the \\ 77th ANNUAL MEETING
}

WASHINGTON, D.C.

APRIL 14-16, 1983 
It is the policy of the American Society of International Law not to take an official position, by resolution or otherwise, upon controversial questions. The views expressed in the addresses, remarks and discussions delivered at its annual meetings and appearing in its printed PROCEEDINGS are those of the individual speakers and are not to be taken as representing the views of the Society.

COPYRIGHT (C) 1985 aY

The American Society of International law

2223 Massachusetts Avenue, N.W.

Washington, D.C. 20008 


\section{PROCEEDINGS OF THE SEVENTY-SEVENTH ANNUAL MEETING OF THE AMERICAN SOCIETY OF INTERNATIONAL LAW}

Chairman of The CommitTeE on the ANNuAl MeEting

Ved P. Nanda
EDITOR OF THE ProceEdings

Ved P. Nanda

AssT. EDITOR: Judith R. Hall

\section{CONTENTS}

\section{Open Forum}

Presiding: Christopher C. Joyner $\ldots \ldots \ldots \ldots \ldots \ldots \ldots \ldots \ldots \ldots \ldots \ldots \ldots \ldots \ldots \ldots \ldots$

Legal Dimensions of the Use of Force in the Falklands War. Alberto R. Coll ......... 1

The Canadian State Immunity Act: A Comparative Perspective. Daniel Turp. .......... 1

The Protection of the Earth-Space Environment: The Utilization of Remote Sensing Satellites

to Implement Human Rights. W. Paul Gormley..................... 2

International Law in the Nuclear Age. Joseph A. Mikus . . . . . . . . . . . . . . . 2

Iran-United States Litigation

Chalrman: Gerald M. Rosberg $\ldots \ldots \ldots \ldots \ldots \ldots \ldots \ldots \ldots \ldots \ldots \ldots \ldots \ldots \ldots \ldots$

REMARKS BY:

Arthur M. Rovine $\ldots \ldots \ldots \ldots \ldots \ldots \ldots \ldots \ldots \ldots \ldots \ldots \ldots \ldots \ldots \ldots \ldots \ldots \ldots \ldots, 3$

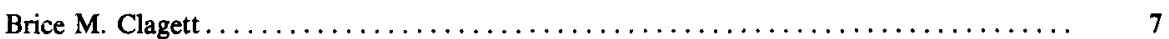

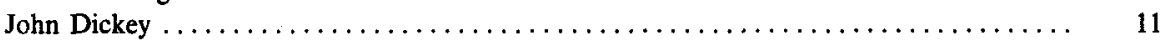

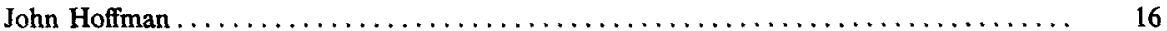

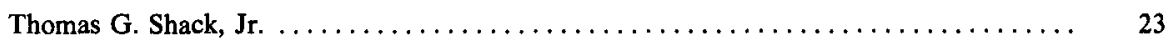

Arthur M. Rovine $\ldots \ldots \ldots \ldots \ldots \ldots \ldots \ldots \ldots \ldots \ldots \ldots \ldots \ldots \ldots \ldots \ldots \ldots$

Armed Force, Peaceful Settlement, and the United Nations Charter: Are There Alternatives to

"A New International Anarchy"?

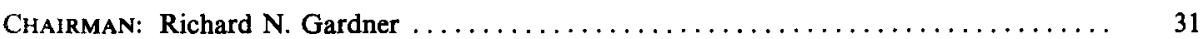

REMARKS BY:

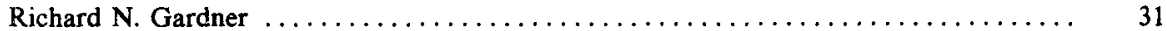

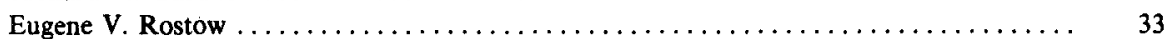

Brian E. Urquhart $\ldots \ldots \ldots \ldots \ldots \ldots \ldots \ldots \ldots \ldots \ldots \ldots \ldots \ldots \ldots \ldots \ldots \ldots \ldots \ldots$

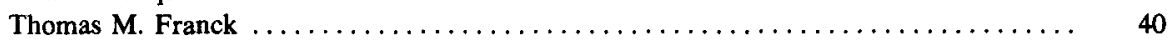

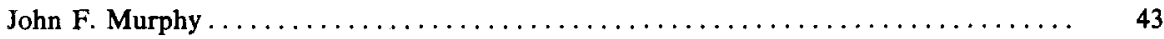

Discussion $\ldots \ldots \ldots \ldots \ldots \ldots \ldots \ldots \ldots \ldots \ldots \ldots \ldots \ldots \ldots \ldots \ldots \ldots \ldots \ldots \ldots \ldots$

Joint Luncheon with the Section of International Law and Practice of the American Bar

Association: Need the World Collapse?

Address by Henry Reuss $\ldots \ldots \ldots \ldots \ldots \ldots \ldots \ldots \ldots \ldots \ldots \ldots \ldots \ldots \ldots \ldots \ldots \ldots$

Transnational Litigation: Discovery Abroad

Chatrman: Bruno A. Ristau $\ldots \ldots \ldots \ldots \ldots \ldots \ldots \ldots \ldots \ldots \ldots \ldots \ldots \ldots \ldots \ldots$

REMARKS BY:

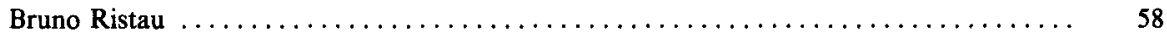

James G. Hergen $\ldots \ldots \ldots \ldots \ldots \ldots \ldots \ldots \ldots \ldots \ldots \ldots \ldots \ldots \ldots \ldots \ldots \ldots \ldots \ldots \ldots, 60$

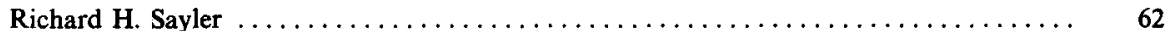

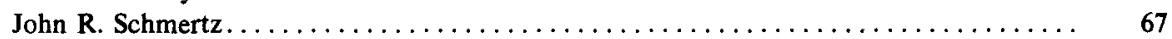

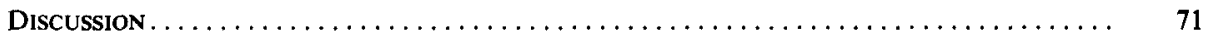

Developments, Restatement II

Chairman: Joseph Modeste Sweeney $\ldots \ldots \ldots \ldots \ldots \ldots \ldots \ldots \ldots \ldots \ldots \ldots \ldots \ldots \ldots$

REMARKS BY:

Joseph Modeste Sweeney $\ldots \ldots \ldots \ldots \ldots \ldots \ldots \ldots \ldots \ldots \ldots \ldots \ldots \ldots \ldots \ldots$ 
Sections of the Revised Draft Restatement Arousing Interest, Comment, and Controversy. Malcolm R. Wilkey $\ldots \ldots \ldots \ldots \ldots \ldots \ldots \ldots \ldots \ldots \ldots \ldots \ldots \ldots \ldots \ldots \ldots, \ldots \ldots$

Act of State and Other Problems with Restatement II (Revised). Malvina Halberstam ...... 80

Some Reflections on the International Law Component of the Foreign Relations Law of the United States: Restatement II (Revised). Christopher Osakwe................ 91

Discussion

International Law in International Diplomacy

Chairman: Francis $O$. Wilcox

REMARKS BY:

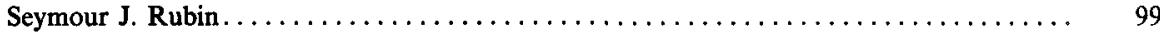

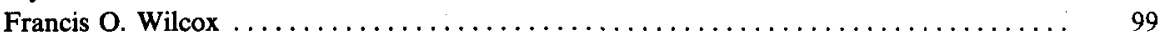

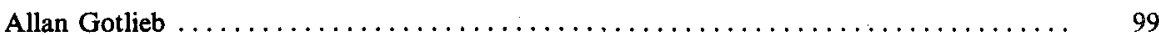

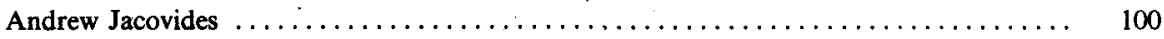

Marcial Perez-Chiriboga $\ldots \ldots \ldots \ldots \ldots \ldots \ldots \ldots \ldots \ldots \ldots \ldots \ldots \ldots \ldots \ldots \ldots \ldots \ldots \ldots, 102$

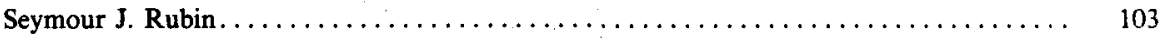

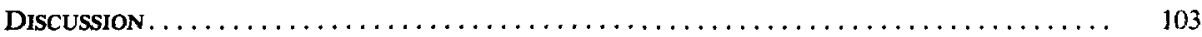

\section{Business Meeting}

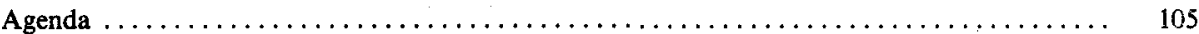

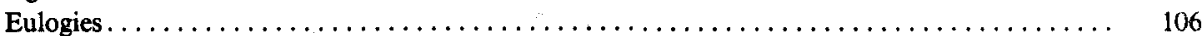

Report of the Executive Director $\ldots \ldots \ldots \ldots \ldots \ldots \ldots \ldots \ldots \ldots \ldots \ldots \ldots \ldots \ldots \ldots \ldots$

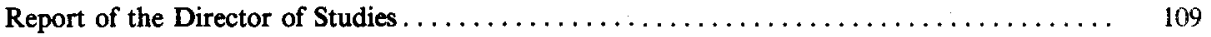

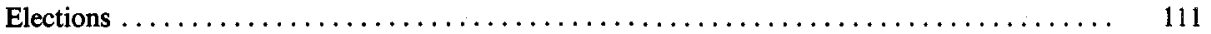

Election of the Nominating Committee $\ldots \ldots \ldots \ldots \ldots \ldots \ldots \ldots \ldots \ldots \ldots \ldots \ldots \ldots \ldots \ldots, 112$

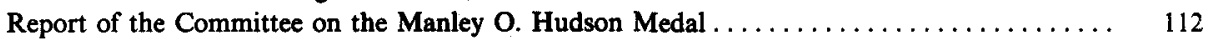

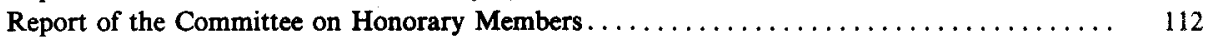

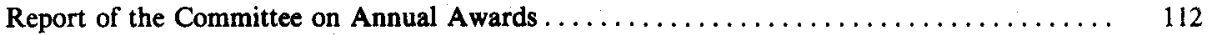

Report of the Committee on Publications of the Department of State

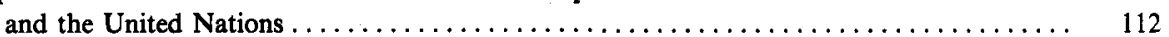

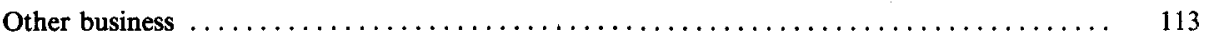

Fair and Unfair Trade in an Interventionist Era

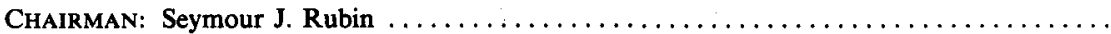

REMARKS BY:

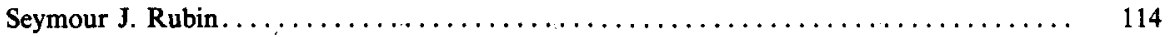

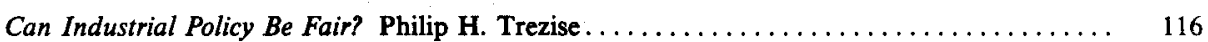

Just Friends: The U.S.-EEC Agricultural Export Subsidies Standoff. Marsha Echols . . . . . . 119

Technology Trade. John H. Barton $\ldots \ldots \ldots \ldots \ldots \ldots \ldots \ldots \ldots \ldots \ldots \ldots \ldots \ldots \ldots \ldots$

Indeterminacy and Legitimation Problems in the Regulation of International Trade. D.K.

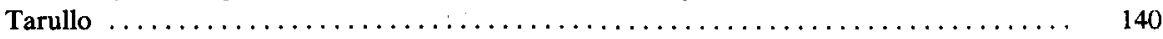

REMARKS BY:

Robert Herzstein $\ldots \ldots \ldots \ldots \ldots \ldots \ldots \ldots \ldots \ldots \ldots \ldots \ldots \ldots \ldots \ldots \ldots \ldots \ldots \ldots \ldots, 146$

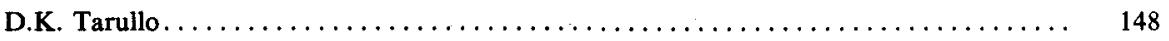

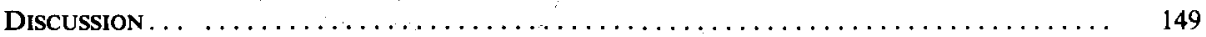

Law of the Sea

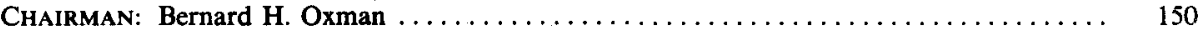

REMARKS BY:

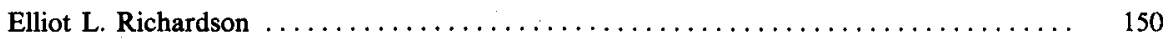

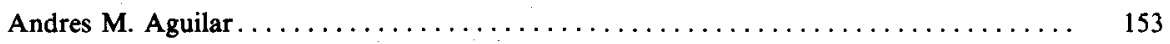

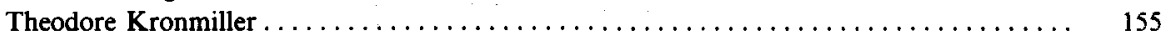

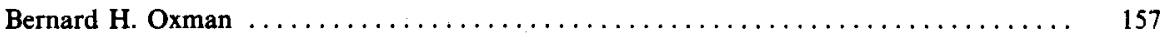

COMMENTARY BY:

Jan Schneider $\ldots \ldots \ldots \ldots \ldots \ldots \ldots \ldots \ldots \ldots \ldots \ldots \ldots \ldots \ldots \ldots \ldots \ldots \ldots \ldots \ldots \ldots$ 
Keith Highet $\ldots \ldots \ldots \ldots \ldots \ldots \ldots \ldots \ldots \ldots \ldots \ldots \ldots \ldots \ldots \ldots, 164$

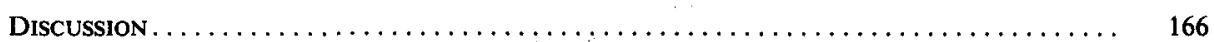

The Control of Violence in a Lebanese Context

Chairman: Covey T. Oliver $\ldots \ldots \ldots \ldots \ldots \ldots \ldots \ldots \ldots \ldots \ldots \ldots \ldots \ldots \ldots, 168$

REMARKS BY:

Covey T. Oliver $\ldots \ldots \ldots \ldots \ldots \ldots \ldots \ldots \ldots \ldots \ldots \ldots \ldots \ldots \ldots \ldots \ldots \ldots \ldots \ldots$

Harold Saunders $\ldots \ldots \ldots \ldots \ldots \ldots \ldots \ldots \ldots \ldots \ldots \ldots \ldots \ldots \ldots \ldots \ldots \ldots \ldots \ldots \ldots$

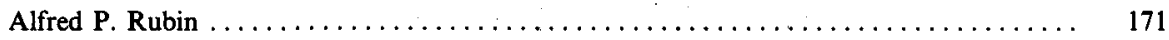

Aggression of Self-Defense in Lebanon in 1982? W. Thomas Mallison . . . . . . . . . . 174

COMMENT BY:

George K. Walker $\ldots \ldots \ldots \ldots \ldots \ldots \ldots \ldots \ldots \ldots \ldots \ldots \ldots \ldots \ldots \ldots \ldots \ldots$

REMARKS BY:

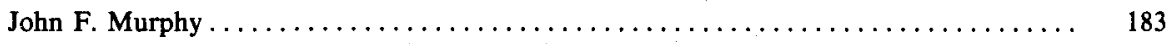

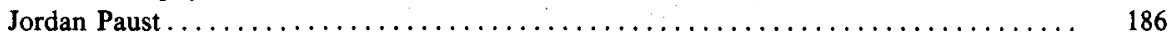

Discussion . . . . . . . . . . . . . . . . . . . . . . . . . . . . . . . . . . 189

Effectiveness of the United Nations

Chalrman: Benjamin B. Ferencz $\ldots \ldots \ldots \ldots \ldots \ldots \ldots \ldots \ldots \ldots \ldots \ldots \ldots \ldots \ldots \ldots \ldots$

REMARKS BY:

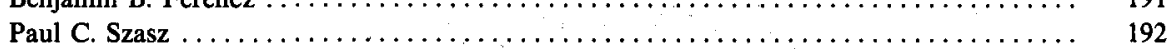

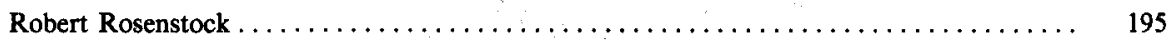

Anthony A. D'Amato $\ldots \ldots \ldots \ldots \ldots \ldots \ldots \ldots \ldots \ldots \ldots \ldots \ldots \ldots \ldots \ldots$

COMMENT BY:
Allan Gerson $\ldots \ldots \ldots \ldots \ldots \ldots \ldots \ldots \ldots \ldots \ldots \ldots \ldots \ldots \ldots \ldots \ldots \ldots \ldots \ldots \ldots \ldots \ldots \ldots \ldots \ldots \ldots$

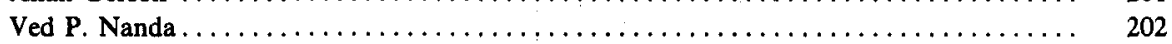

SUMMARY BY:
$\quad$ Benjamin B. Ferencz $\ldots \ldots \ldots \ldots \ldots \ldots \ldots \ldots \ldots \ldots \ldots \ldots \ldots \ldots \ldots \ldots \ldots \ldots \ldots \ldots \ldots \ldots 203$

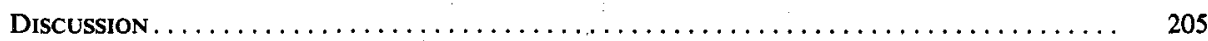

Humanitarian Law

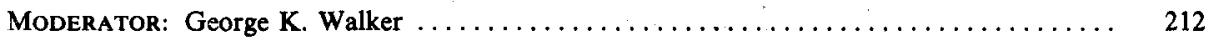

Grotius: His Relevance to Present Day Law of Armed Conflict. Frits Kalshoven. . . . . . . 213

Problems of the Law of Armed Conflict in Lebanon

REMARKS BY:

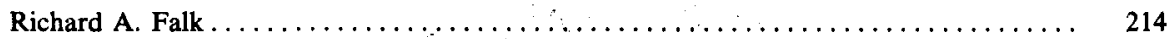

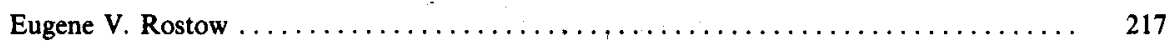

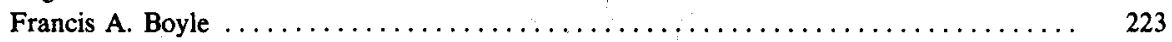

COMMENTS BY:

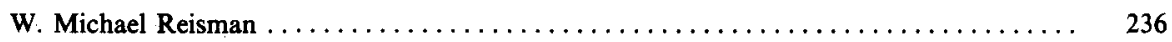

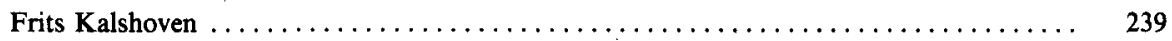

REMARKS BY:

Robbie Sabel . . . . . . . . . . . . . . . . . . . . . . . . . . . . . 240

Extraterritorial Application of U.S. Export Controls-The Siberian Pipeline

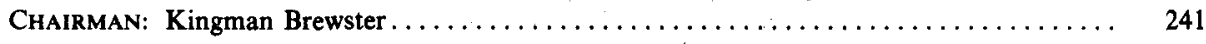

REMARKS BY:

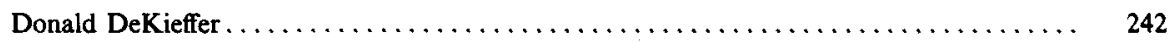

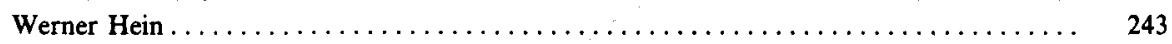

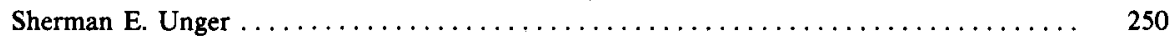

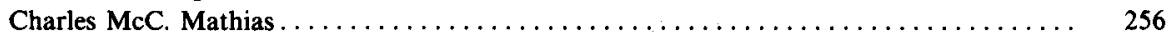

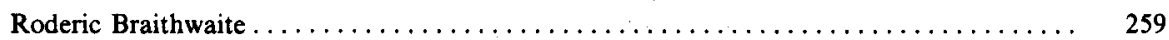

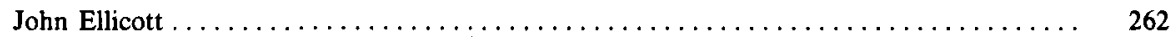

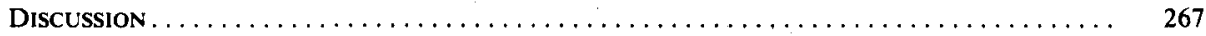


Settlement in the Middle East: What Would It Look Like and Can We Get There from Here?

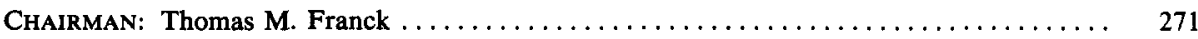

Round TABLE Discussion $\ldots \ldots \ldots \ldots \ldots \ldots \ldots \ldots \ldots \ldots \ldots \ldots \ldots \ldots \ldots \ldots \ldots \ldots \ldots \ldots$

DISCUSSION FROM THE FLOOR $\ldots \ldots \ldots \ldots \ldots \ldots \ldots \ldots \ldots \ldots \ldots \ldots \ldots \ldots \ldots \ldots \ldots$

Can the GATT Resolve International Trade Disputes?

Chatrman: William Diebold $\ldots \ldots \ldots \ldots \ldots \ldots \ldots \ldots \ldots \ldots \ldots \ldots \ldots \ldots \ldots \ldots$

Introductory Remarks by: William Diebold.

REMARKS BY:

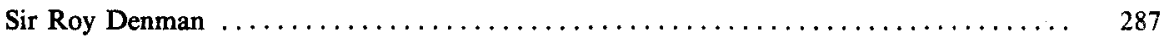

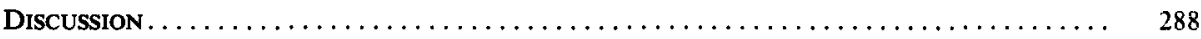

REMARKS BY:

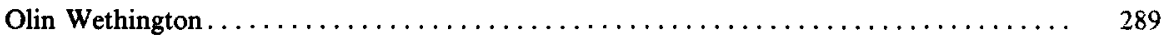

Robert C. Cassidy, Jr. . . . . . . . . . . . . . . . . . . . . . . . . . . . . . . 289

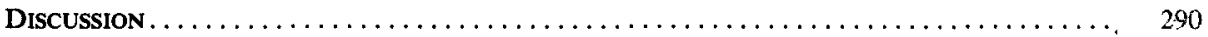

Legal Principles and Practices Relating to Private Foreign Investment

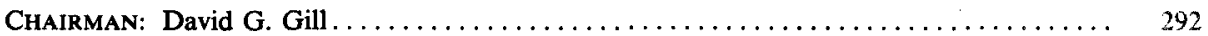

REMARKS BY:

David G. Gill $\ldots \ldots \ldots \ldots \ldots \ldots \ldots \ldots \ldots \ldots \ldots \ldots \ldots \ldots \ldots \ldots \ldots \ldots \ldots \ldots \ldots \ldots \ldots$

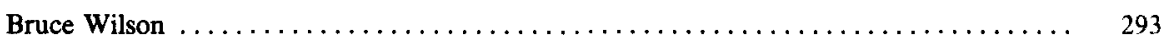

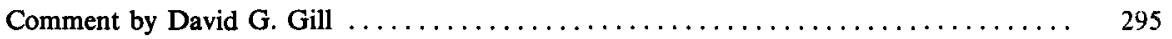

REMARKS BY:

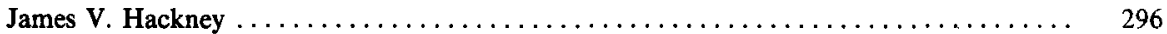

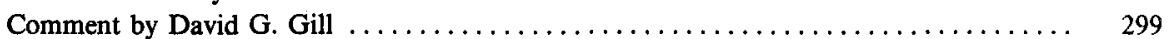

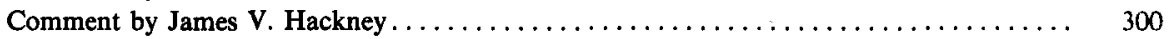

REMARKS BY:

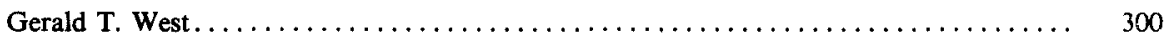

Heribert Golsong $\ldots \ldots \ldots \ldots \ldots \ldots \ldots \ldots \ldots \ldots \ldots \ldots \ldots \ldots \ldots \ldots \ldots \ldots \ldots \ldots, 303$

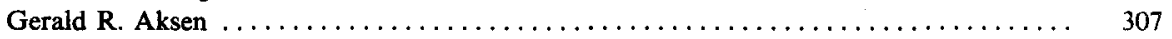

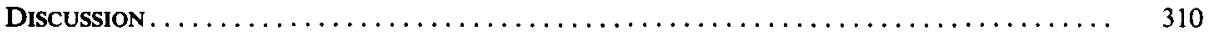

Restructuring Sovereign Debt-Will There be New International Law and Institutions?

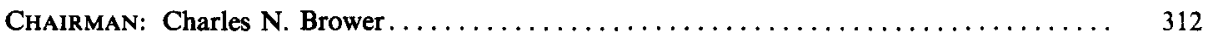

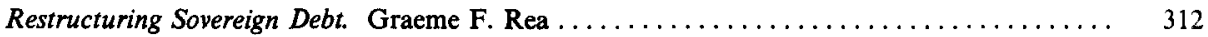

International Debt Problems: The Role of the World Bank. George R. Hoguet ....... 317

Restructuring Sovereign Debt: The Perspective of the Sovereign Debtor. Christine D.

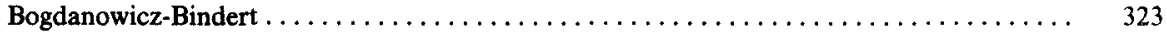

AdDitional REMARKs $\ldots \ldots \ldots \ldots \ldots \ldots \ldots \ldots \ldots \ldots \ldots \ldots \ldots \ldots \ldots \ldots \ldots \ldots$

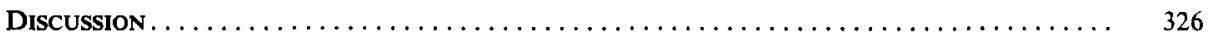

COMMENT BY:

Cecil Hunt $\ldots \ldots \ldots \ldots \ldots \ldots \ldots \ldots \ldots \ldots \ldots \ldots \ldots \ldots \ldots \ldots \ldots \ldots \ldots \ldots \ldots$

Adjudication of Foreign Expropriations in U.S. Courts

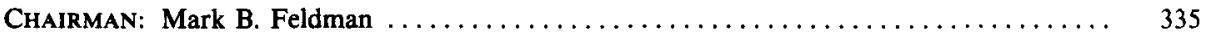

REMARKS BY:

Mark B. Feldman . . . . . . . . . . . . . . . . $\ldots \ldots \ldots \ldots \ldots \ldots \ldots \ldots \ldots$

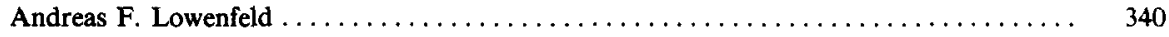

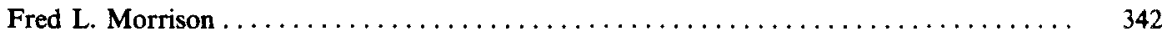

Victor Rabinowitz $\ldots \ldots \ldots \ldots \ldots \ldots \ldots \ldots \ldots \ldots \ldots \ldots \ldots \ldots \ldots \ldots$

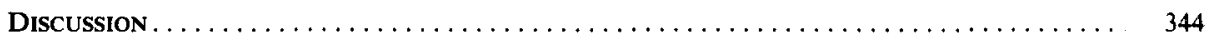




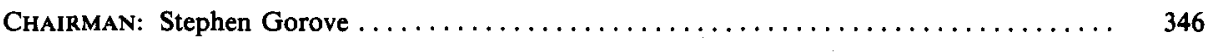

INTRODUCTION BY:

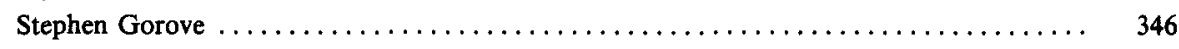

Space Telecommunications-Issues and Policies: Role of the United Nations. Nandasiri

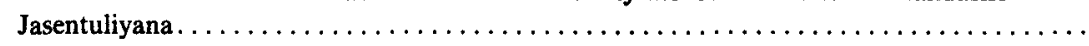

The Plenipotentiary Conference of the International Telecommunication Union, Nairobi, 1982:

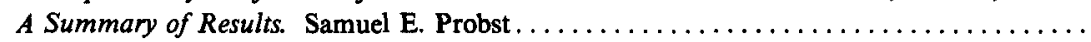

REMARKS BY:

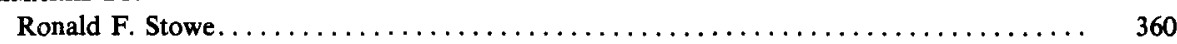

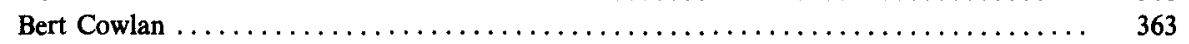

COMMENTS BY:

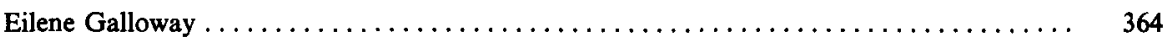

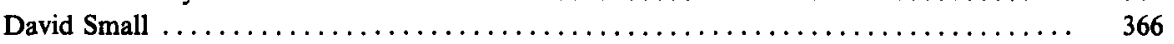

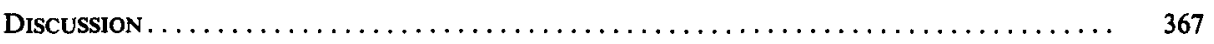

\section{Annual Dinner}

Extraterritoriality and Conflicts of Jurisdiction. Address by Kenneth W. Dam . . . . . . 370

Human Rights-Effective Remedies

ChaIRMAN: Thomas Buergenthal $\ldots \ldots \ldots \ldots \ldots \ldots \ldots \ldots \ldots \ldots \ldots \ldots \ldots \ldots \ldots \ldots$

International Measures Against Arbitrary Killings by Governments. David Weissbrodt ...... 378

REMARKS BY:

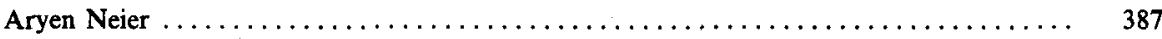

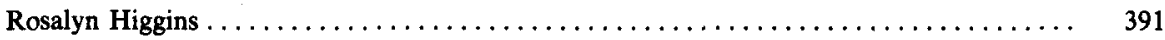

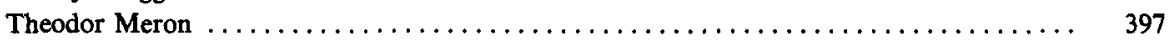

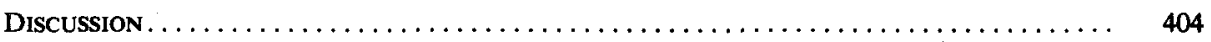

Philip C. Jessup International Law Moot Court Competition $\ldots \ldots \ldots \ldots \ldots \ldots \ldots \ldots \ldots \ldots$

Ten Years after Stockholm-International Environmental Law

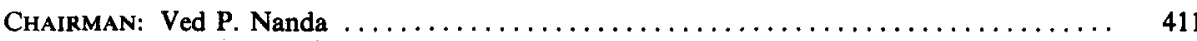

International Environmental Law and the Work of the International Law Commission.

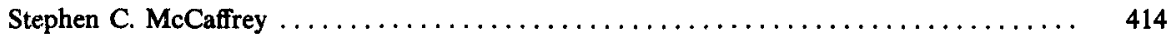

The Struggle for the Internationally Shared Environment: The United States Abdicates Its

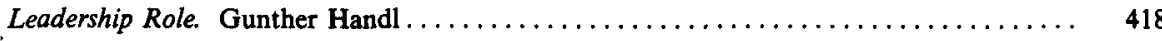

Environment and Development. Howard Taubenfeld $\ldots \ldots \ldots \ldots \ldots \ldots \ldots \ldots \ldots \ldots, 423$

COMMENTS BY:

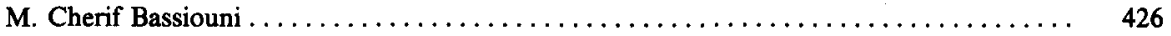

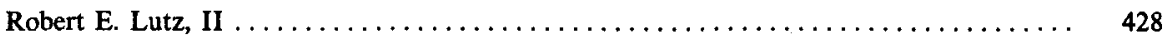

George William Sherk $\ldots \ldots \ldots \ldots \ldots \ldots \ldots \ldots \ldots \ldots \ldots \ldots \ldots \ldots \ldots \ldots \ldots . \ldots \ldots$

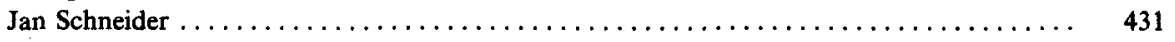

Discussion $\ldots \ldots \ldots \ldots \ldots \ldots \ldots \ldots \ldots \ldots \ldots \ldots \ldots \ldots \ldots \ldots \ldots \ldots \ldots \ldots \ldots \ldots$

Appendices

Association of Student International Law Societies: The Executive Secretary's Report ......

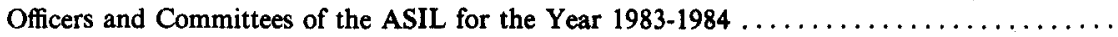

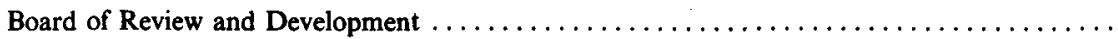
Report of the Ad Hoc Committee to Review the Organization and Activities of the American

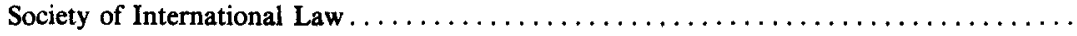

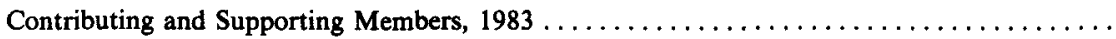

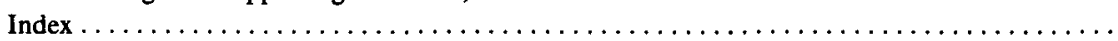

